\title{
Giant magnetoresistance in NiFe-Ag granular alloys
}

\author{
F. Badía, A. Labarta, and X. Batlle \\ Departament Física Fonamental, Universitat de Barcelona, Diagonal 647, 08028 Barcelona, Spain \\ M. L. Watson \\ Centre for Data Storage Materials, Coventry University, Priory Street, Coventry CVI 5FB, United Kingdom
}

\begin{abstract}
Some FeNi-Ag granular films of composition $\mathrm{Fe}_{11.43} \mathrm{Ni}_{6.35} \mathrm{Ag}_{82.22}$ (sample $\mathrm{A}$ ) and $\mathrm{Fe}_{7.62} \mathrm{Ni}_{16.4} \mathrm{Ag}_{75.98}$ (B) were prepared by using rf magnetron sputtering, and once deposited were rapidly annealed at 600,650 , and $750^{\circ} \mathrm{C}$. All samples displayed giant magnetoresistance. The zero-field-cooled and field-cooled processes evidence the segregation of ferromagnetic particles with a broad size distribution. The temperature and magnetic field dependence of the resistance is analyzed. The magnetoresistance follows a $H^{n}$ law at high fields and it decays from its maximum value with a $T^{m}$ behavior, with $m$ approaching 1 at high fields.
\end{abstract}

\section{INTRODUCTION}

The discovery of giant magnetoresistance (GMR) effects in a variety of antiferromagnetically coupled transition-metal multilayers ${ }^{1}$ has opened a new amazing research field not only from the fundamental point of view but also from the technological one. Recently, this extraordinary effect has also been found in granular alloys ${ }^{2}$ consisting of a distribution of nonaligned nanocrystalline ferromagnetic particles embedded in a nonmagnetic metallic matrix. In both kinds of systems, the resistivity strongly drops as the magnetic field orients the magnetic moments. Concerning theoretical explanations, both the existence of a spin-dependent potential scattering either at the interfaces or in the bulk of ferromagnetic layers (or particles) and the role of the unfilled $d$ bands of the transition metal constituent (through an asymmetric density of states for majority- and minority-spin $d$ bands) $)^{3,4}$ have been taken into account in order to correlate GMR with the microscopic parameters. The magnitude of GMR has been found to be a sensitive function of both the size of the ferromagnetic particles and the concentration of the ferromagnetic material in the alloy. The former effect is postulated to be due to the existence of an optimum particle size, determined by the conduction electron mean free path or spin diffusion length. Larger particles result in a reduction of GMR as a result of the decrease in particle surface-tovolume ratio. ${ }^{3}$ The latter effect is believed to be due to the onset of percolation, which acts to couple the particles ferromagnetically. ${ }^{1-3}$ We present in this article the temperature and magnetic field dependence of the resistance of $\mathrm{NiFe}-\mathrm{Ag}$ granular alloys presenting GMR. ${ }^{5}$

\section{EXPERIMENT}

$\mathrm{Ag}-\mathrm{Ni}-\mathrm{Fe}$ films of thickness $200-300 \mathrm{~nm}$ were rf sputtered onto glass microscope slides using a Nordico 2000 sputtering system. The base pressure was less than $2 \times 10^{-7}$ Torr, the sputtering pressure was 8 mTorr of argon and the sputtering power was $300 \mathrm{~W}$. The target used consisted of a 4 in. $\mathrm{Ag} \mathrm{(99.999 \% )} \mathrm{disc} \mathrm{onto} \mathrm{which} \mathrm{were} \mathrm{placed} \mathrm{Ni}_{80} \mathrm{Fe}_{20}$ and $\mathrm{Fe} 0.25 \mathrm{~cm}^{2}$ squares arranged in a mosaic pattern. In order to promote post-deposition phase segregation and magnetic particle growth, strips of about $7 \mathrm{~mm}$ wide were rapidly thermally annealed in a custom built vacuum system. Three an- nealing temperatures were investigated: 600,650 , and $750^{\circ} \mathrm{C}$, and these were reached in $20 \mathrm{~s}, 2 \mathrm{~min}$, and $3 \mathrm{~min}$, respectively. Resistance and magnetoresistance (MR) of all samples were measured by an ac four-point probe technique in the temperature range $20-300 \mathrm{~K}$ and in magnetic fields up to $12 \mathrm{kOe}$. The relative geometry among the film plane, the electrical current, and the magnetic field was set by three ways: (a) the electrical current and the magnetic field are parallel to the film plane (parallel geometry); (b) the in-plane magnetic field is perpendicular to the electrical current (transversal geometry); and (c) the magnetic field is perpendicular to both the electrical current and the film plane (perpendicular geometry). The zero-field-cooled (ZFC) and fieldcooled (FC) processes at low fields and the magnetization curves at $5 \mathrm{~K}$ up to $55 \mathrm{kOe}$ were carried out by applying the magnetic field along the film plane using a superconducting quantum interference device magnetometer.

\section{RESULTS AND DISCUSSION}

The structure of some of the thin films $(d \approx 50 \mathrm{~nm})$ were investigated by transmission electron microscopy (TEM) in a modified JEOL 2000 electron microscope. Films were deposited onto Si substrates into which a SiN covered window had been etched and were found to have a strong $\langle 111\rangle$ texture. A number of films were also investigated by using a Philips $\mathrm{x}$-ray diffraction (XRD) system. This confirmed the strong $\langle 111\rangle$ texturing but in neither the TEM nor the XRD was any clear evidence of phase segregation of the $\mathrm{Ni}$ or $\mathrm{Fe}$ from the $\mathrm{Ag}$ matrix. Magnetic and transport properties were measured on films which had composition $\mathrm{Fe}_{11.43} \mathrm{Ni}_{6.35} \mathrm{Ag}_{82.22}$ (sample A) and $\mathrm{Fe}_{7.62} \mathrm{Ni}_{16.4} \mathrm{Ag}_{75.98}$ (sample B). As both samples were rapidly annealed at 600,650 , and $750^{\circ} \mathrm{C}$, we will refer to them as $\mathrm{A}$ (as cast), $\mathrm{A}(600), \mathrm{A}(650), \mathrm{A}(750), \mathrm{B}$ (as cast), $\mathrm{B}(600), \mathrm{B}(650)$, and $\mathrm{B}(750)$, respectively.

Figure 1 shows the ZFC-FC processes for sample A(650) measured at $100 \mathrm{Oe}$. The ZFC displays a broad maximum at $T_{M} \approx 22 \mathrm{~K}$, suggesting the existence of a broad size distribution of ferromagnetic particles. As magnetic irreversibility persists up to high temperature, we expect very large particles to be present in the sample. We plot in Fig. 2 the magnetization curve of the same sample at $5 \mathrm{~K}$. A detail of 


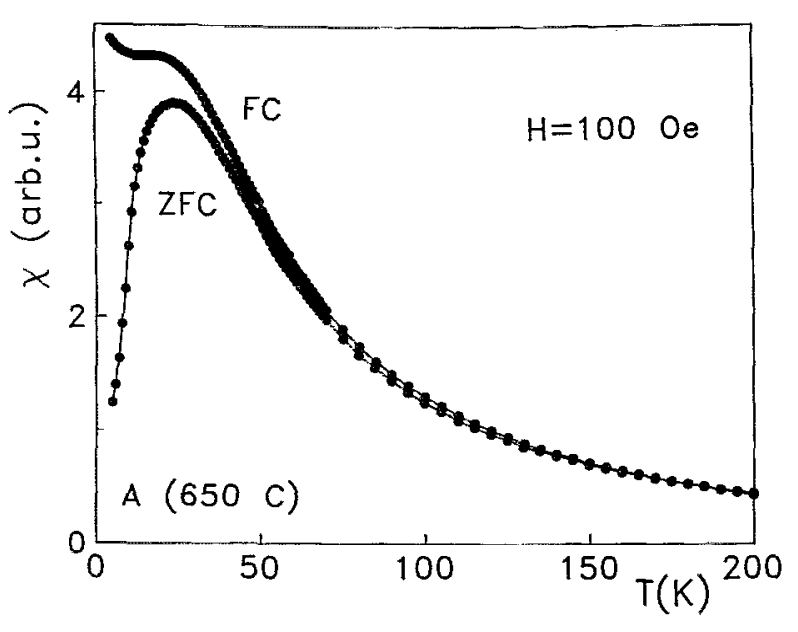

FIG. 1. ZFC and FC processes measured at 100 Oe for sample A(650).

the hysteresis circle is displayed in the inset, showing that the coercive field is small (about 150 Oe).

The temperature dependence of the ratio $-R_{M}(T, H) / R(T, H=0) \quad\left[\right.$ with $\quad R_{M}(T, H)=R(T, H=0)$ $-R(T, H)]$ in the parallel geometry for samples $\mathrm{A}(650)$ and $\mathrm{B}(650)$ are plotted in Figs. 3 and 4, respectively. All the rest of the samples display very similar experimental features. The maximum MR values are obtained for samples B(650) and $A(650)$, suggesting that the optimum annealing temperature is about $650^{\circ} \mathrm{C}$ in this $\mathrm{Ag}$ compositional range. MR is larger for sample $B(650)$ than for sample $A(650)$ because of the larger amount of ferromagnetic entities. As-cast samples display smaller MR than annealed samples due to the segregation of ferromagnetic particles in the latter. Results concerning the rest of annealing temperatures and other Ag compositions will be published elsewhere. Figures 3 and 4 evidence that MR is largely susceptible at low fields. We have also detected that MR is more susceptible in the inplane geometries than in the perpendicular geometry, which is only due to the demagnetizing field (and it is not associ-

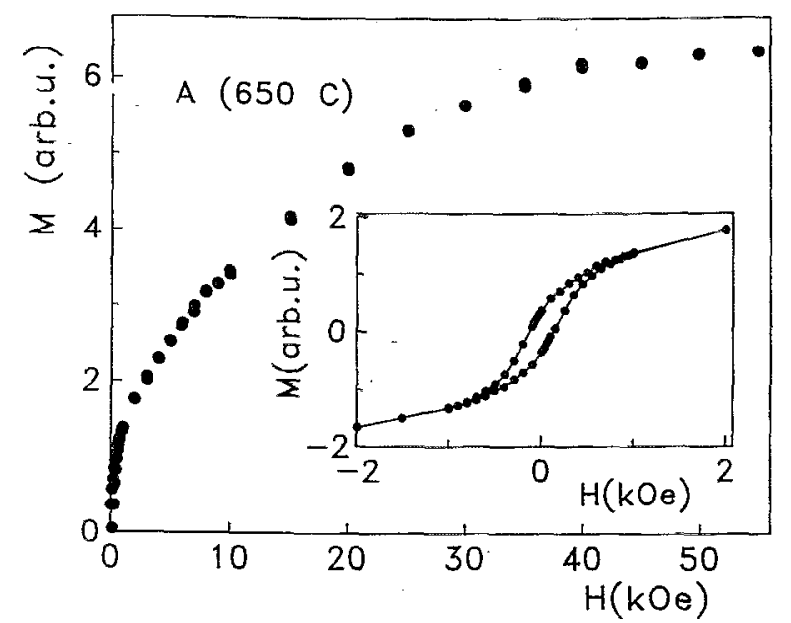

FIG. 2. Isothermal magnetization curve at $5 \mathrm{~K}$ for sample $\mathrm{A}(650)$. Inset: detail of the hysteresis cycle at $5 \mathrm{~K}$ for the same sample.

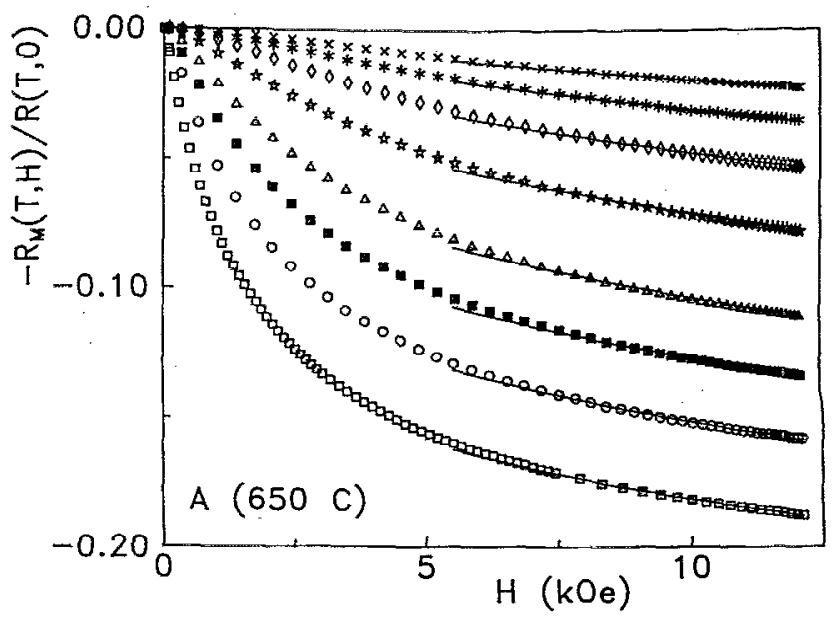

FIG. 3. $-R_{M}(T / H) / R(T, H=0)$ as a function of $H$ at different temperatures for sample $A(650)$ in the parallel geometry. Solids lines indicate the best fit of the data to the $H^{n}$ law. Temperatures: (口) $21.6 \mathrm{~K}$; (O) $46.1 \mathrm{~K}$; (O) 73.6 $\mathrm{K} ;(\triangle) 102 \mathrm{~K} ;(\diamond) 149.2 \mathrm{~K} ;(\diamond) 196.4 \mathrm{~K} ;\left(^{*}\right) 245 \mathrm{~K} ;(\times) 290 \mathrm{~K}$.

ated with an intrinsic in-plane magnetocrystalline anisotropy) because there is no difference between the MR in the parallel and transversal geometry. All measurements have been recorded with increasing and decreasing field, and we observe a slight irreversibility at low temperatures below the coercive field.

We have analyzed the temperature dependence of the MR as Mattson et al. ${ }^{6}$ by defining the MR as $R_{M}(T, H)=R(T, H=0)-R(T, H)$, where $R(T, H)$ is the resistance measured at a temperature $T$ and in an applied field $H$. The total resistance at $T$ and $H$ is assumed to be given by $R(T, H)=R_{0}+R_{s d}(T)+R_{M}(T, H)$, where $R_{0}$ is the resistance due to defects, $R_{s d}(T)$ is the temperature dependent resistance due to phonons and magnons. We show in Fig. 5 the temperature dependence of $R_{M}(T, H)$ at different fields for sample $\mathrm{A}(650) . R_{M}(T, H)$ displays a monotonic increase

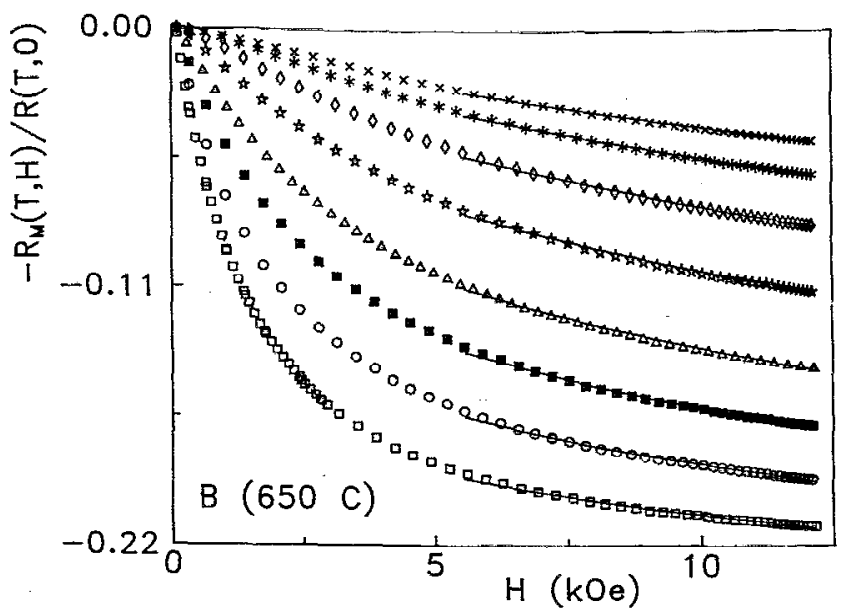

FIG. 4. $-R_{M}(T / H) / R(T, H=0)$ as a function of $H$ at different temperatures for sample $B(650)$ in the parallel geometry. Solids lines indicate the best fit of the data to the $H^{n}$ law. Temperatures: (口) $21.5 \mathrm{~K}$; (O) $45.9 \mathrm{~K}$; (๑) 73.7

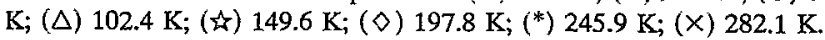




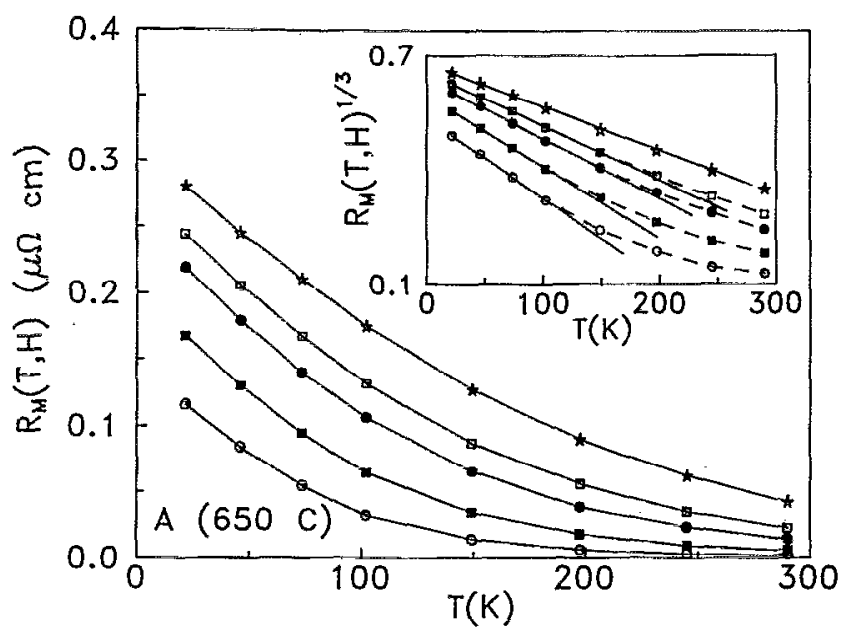

FIG. 5. $R_{M}(T, H)$ vs $T$ at various fields for sample $\mathrm{A}(650)$. Magnetic fields: (O) $1 \mathrm{kOe;} \mathrm{(D)} 2$ kOe; (O) $4 \mathrm{kOe}$; (口) $6 \mathrm{kOe;} \mathrm{(St)} 12.1$ kOe. Inset: $R_{M}(T, H)^{1 / 3}$ vs $T$ at the same fields for the same sample.

as temperature goes down, which is in agreement with the progressive blocking of the ferromagnetic particles (Fig. 1). We plot in the inset of Fig. 5 the temperature dependence of $R_{M}(T, H)^{1 / 3}$ at various fields for the same sample. We notice that $R_{M}(T, H)^{1 / 3}$ is perfectly linear with $T$ in the whole temperature range $20-290 \mathrm{~K}$ when the magnetic field is the maximum available in our experimental setup $\left(H_{\max }=12\right.$ $\mathrm{kOe}$ ), and that the linear law $R_{M}(T, H)^{1 / 3}$ versus $T$ is followed in a smaller temperature range as we reduce the magnetic field. The same $1 / 3$ exponent and temperature dependence of $R_{M}(T, H)$ is found for sample $\mathrm{B}(650)$. If we extrapolate the data at $T=0$, we obtain the $R_{M}(T=0, H)$ and we may define $\Delta R_{M}=R_{M}(T=0, H)-R_{M}(T, H)$. The log-log plot of $\Delta R_{M}$ versus $T$ is displayed in Fig. 6 for sample $\mathrm{A}(650)$ at 2 and $12.1 \mathrm{kOe}$. The slope of the plot yields the exponent $m$ in the relationship $\Delta R_{M} \approx T^{m}$, and this power

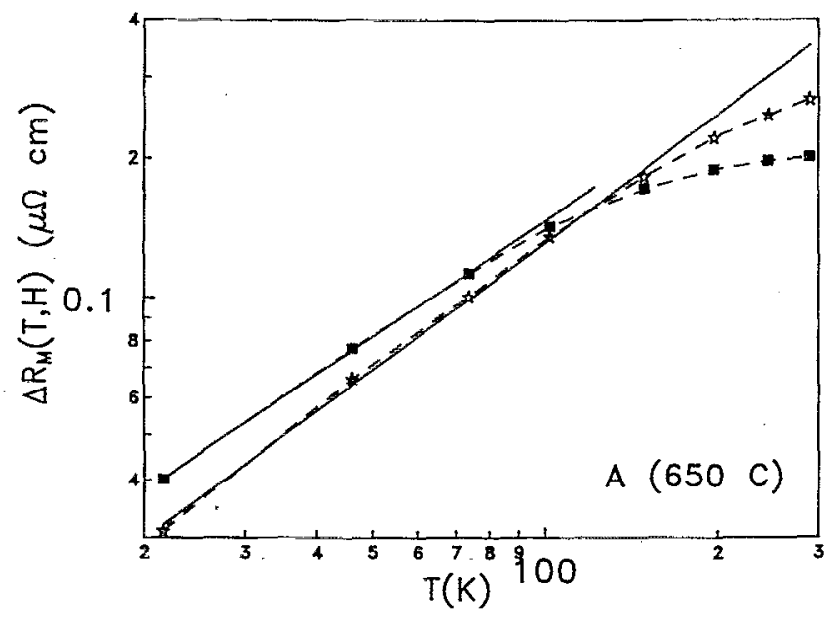

FIG. 6. Log-log plot of the temperature dependence of $\Delta R_{M}=R_{M}(T=0, H)-R_{M}(T, H)$, at $(\square) 2 \mathrm{kOe}$ and at (价) $12.1 \mathrm{kOe}$ for the sample $A(650)$. Solid lines correspond to the best fit of the data to a $T^{m}$ law. law gives us an idea of the underlying scattering mechanism. It is evidenced that the temperature range in which the power law is accomplished increases with magnetic field (as expected, since the MR saturates at large fields). The exponent $m$ slightly increases with $H$ and seems to tend to about 1 , which is smaller than the $T^{3 / 2}$ and $T^{2}$ laws found by Mattson et al. ${ }^{6}$ in $\mathrm{Fe} / \mathrm{Cr}$ multilayers. These behaviors are attributed to the thermal excitation of magnons. The temperature dependence of the MR of granular materials is complicated by there being a distribution of particle sizes and therefore blocking processes. We might tentatively attribute the temperature dependence of $\Delta R_{M}$ at high fields to the thermal excitation of magnons with a smaller exponent in the $T^{m}$ law due to the reduction of the magnelic system dimensionality.

Concerning the field dependence of the $M R$, we have observed that $R_{M}(T, H) / R(T, 0)$ follows a $H^{n}$ behavior at high fields (above about $6000 \mathrm{Oe}$ ), as was found by Nigam et al. ${ }^{7}$ in $\mathrm{Au}_{87} \mathrm{Fe}_{13}$ cluster glass. Solids lines in Figs. 3 and 4 indicate the best fit of the data to the $H^{n}$ law. The $n$-exponent monotonically increases with temperature, ranging from 0.18 at $21.6 \mathrm{~K}$ to 0.86 at $290 \mathrm{~K}$ for samples $\mathrm{A}(650)$, and from 0.13 at $21.5 \mathrm{~K}$ to 0.76 at $282.1 \mathrm{~K}$ for sample $\mathrm{B}(650)$. The error in $n$ is about 0.02 . This monotonic temperature behavior evidences the progressive blocking of the ferromagnetic particles, without being a freezing state corresponding to a spin glass behavior. Also, $n$ is always smaller than the $n=2$ value expected for a pure paramagnetic state, ${ }^{7}$ signaling that magnetic correlations persist even at room temperature and/or larger particles are still blocked at this temperature, since the size distribution of ferromagnetic particles seems to be very broad (see Fig. 1).

\section{ACKNOWLEDGMENTS}

We gratefully acknowledge the assistance of Professor J. Chapman and Dr. A. Johnston of the University of Glasgow who conducted the TEM investigations.

${ }^{I}$ M. N. Baibich et al., Phys. Rev. Lett. 61, 2472 (1988); G. Binach, P. Grunberg, F. Saurenbach, and W. Zinn, Phys. Rev. B 39, 4828 (1989); W. P. Pratt, S. F. Lee, J. M. Slaughter, R. Loloee, P. A. Schroeder, and J. Bass, Phys. Rev. Lett. 66, 3060 (1991).

${ }^{2}$ A, E. Berkowitz, J. R. Mitchell, M. I. Carey, A. P. Young, S. Zhang, F. E. Spada, F. T. Parker, H. Hutten, and G. Thomas, Phys. Rev. Lett. 68, 3745 (1992); J. Q. Xiao, J. S. Jiang, and C. L. Chien, ibid. 68, 3749 (1992); J. A. Barnard, A. Waknis, M. Tan, E. Haftek, M. R. Parker, and M. L. Watson, J. Magn. Magn. Mater. 114, L230 (1992).

${ }^{3}$ S. Zhang, Appl. Phys. Lett. 61, 1855 (1992); S. Zhang and P. M. Levy, J. Appl. Pliys. 73, 5316 (1993).

${ }^{4}$ L. Xing and Y. C. Chang, Phys. Rev. B 48, 4156 (1993); L. Xing, Y. C. Chang, M. B. Salomon, D. M. Frankel, J. Shi, and J. P. Lu, ibid. 48, 6728 (1993).

5 J. S. Jiang, J. Q. Xiao, and C. L. Chien, Appl. Phys. Lett. 61, 2362 (1992); M. L. Watson, J. A. Barnard, S. Hossain, and M. R. Parker, J. Appl. Phys. 73, 5506 (1993).

${ }^{6}$ I. A. Campbell, A. Fert, and O. Jaoul, J. Phys. C 3, S95 (1970); P. P. Freitas and L. Berger, J. Magn. Magn. Mater. 54-57 (1986); M. A. M. Gijs and M. Okada, ibid. 113, 105 (1992); J. E. Mattson, M. E. Brubaker, C. H. Sowers, M. Conover, Z. Qiu, and S. D. Bader, Phys. Rev. B 44, 9378 (1991).

${ }^{7}$ A. K. Nigam, S. Radha, and G. Chandra, J. Phys.: Condens. Matt. 5, 9197 (1993). 